\title{
Erratum: Edge phase transitions of the tricritical Potts model in two dimensions [Phys. Rev. E 71, 026109 (2005)]
}

Youjin Deng and Henk W. J. Blöte

Q (Received 2 May 2019; published 26 June 2019)

DOI: 10.1103/PhysRevE.99.069904

We strongly suspect that the results for the ratio $Q_{\text {sf }}$ in Sec. III B 4 were actually obtained from the bulk Binder ratio but mislabeled as surface results. Although the old data are no longer available, an analysis of newly computed data is provided in Ref. [1].

[1] H. W. J. Blöte and Y. Deng, Phys. Rev. E 99, 062133 (2019). 